\title{
Application of quantitative precipitation forecasting and precipitation ensemble prediction for hydrological forecasting
}

\author{
PENG TAO ${ }^{1,2}$, SHEN TIE-YUAN ${ }^{1}$, YIN ZHI-YUAN ${ }^{1}$ \& WANG JUN-CHAO ${ }^{1}$ \\ 1 Hubei Key Laboratory for Heavy Rain Monitoring and Warning Research, Institute of Heavy Rain, CMA, Wuhan \\ 430205, China \\ pt mail@sohu.com \\ 2 State Key Laboratory of Severe Weather, Chinese Academy of Meteorological Sciences, Beijing 100081, China
}

\begin{abstract}
The precipitation in the forecast period influences flood forecasting precision, due to the uncertainty of the input to the hydrological model. Taking the ZhangHe basin as the example, the research adopts the precipitation forecast and ensemble precipitation forecast product of the AREM model, uses the Xin Anjiang hydrological model, and tests the flood forecasts. The results show that the flood forecast result can be clearly improved when considering precipitation during the forecast period. Hydrological forecast based on Ensemble Precipitation prediction gives better hydrological forecast information, better satisfying the need for risk information for flood prevention and disaster reduction, and has broad development opportunities.
\end{abstract}

Key words AREM model; quantitative precipitation forecast; precipitation ensemble prediction; Xin Anjiang model; flood forecast

\section{INTRODUCTION}

Rainstorms are the major type of severe weather in China. Floods from rainstorms often bring huge losses of life and property. Precipitation in the foreseeable period has a direct impact on the accuracy of flood forecasting. The longer the period foreseen, the greater the impact on the flood forecast results. Therefore, the coupling technology for precipitation in the foreseeable period and flood forecasting has gradually been considered by many hydrologists and meteorologists in recent years (Yang Wen-fa et al. 2006; Cui Chun-guang and Peng Tao 2010). At present, with the rapid development of numerical prediction theory and methods, numerical prediction is becoming a scientific method to obtain the location, timing and quantity of rainstorm precipitation, and provide strong support for estimates of rainfall in the foreseeable period in hydrological models. However, due to the uncertainty of the numerical model forecast precipitation, its intensity and the position of heavy precipitation has deviations from the actual precipitation, so is bound to cause error in the hydrological forecast. With the development of short-term precipitation ensemble prediction technology, improved hydrological forecasts have been obtained in other countries, and there are high expectations (Zhang Hong-gang and Guo Shen-lian 2006; Li Jun 2007; Li Yan 2008). Precipitation ensemble prediction products have higher accuracy and the calculation stability is good relative to single model forecasts, so precipitation ensemble prediction was introduced into the hydrological forecast, and can form a meteorological and hydrological forecasting chain, so improve the science of flood control operations and decision-making, and the precision of hydrological forecasts. In a case study of the ZhangHe Reservoir catchment, in Hubei province, China, during the flood season in 2008, the precipitation and ensemble prediction products of a numerical model were used with a hydrological model; we applied to give a quantitative precipitation forecast and its ensemble forecast in the flood forecast.

\section{SUMMARY OF RESEARCH AREA}

The ZhangHe originates from the southern foot of Jingshan, Nanzhang County, Hubei. It flows $202 \mathrm{~km}$ through counties and cities as Baokang, Jingmen and Dangyang. The basin is a long northwest/southeast oriented strip with an average length of $100 \mathrm{~km}$ and width of $30 \mathrm{~km}$, and a drainage area of $2980 \mathrm{~km}^{2}$. ZhangHe Reservoir, located in Jingmen city, has a catchment area of $2212 \mathrm{~km}^{2}$; its total storage capacity is 2.03 billion $\mathrm{m}^{3}$ (Fig. 1).

The basin belongs to the Middle Yangtze River subtropical monsoon area. The annual average rainfall is $1003.6 \mathrm{~mm}$. Rainstorms happen frequently with high intensity. Floods fluctuate 


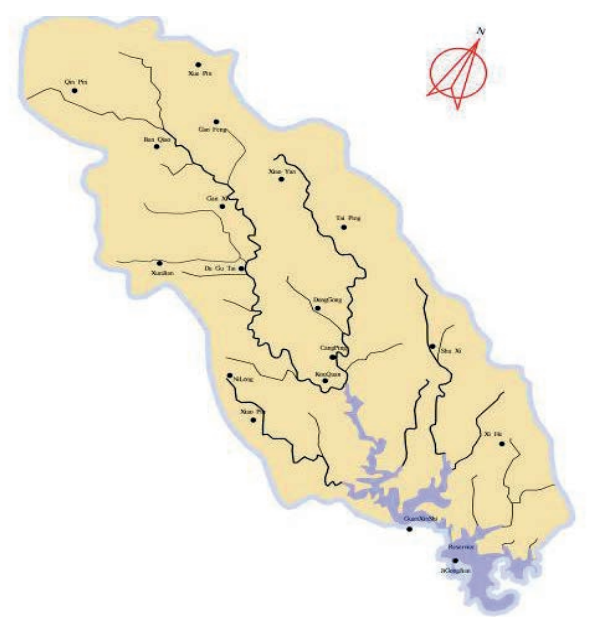

Fig. 1 The sketch map for ZhangHe Catchment.

greatly; their rise time is short. The hydrological monitoring network in the basin is homogeneous (Fig. 1). These may help the study of short-term flood forecast.

\section{TEST OF PRECIPITATION FORECAST AND ENSEMBLE FORECAST}

\subsection{Selection of numerical model}

The meso-scale numerical model AREM (Advanced Regional Eta Model) with high-resolution and a three-dimensional variational assimilation system was developed by the Institute of Atmospheric Physics, Chinese Academy of Sciences and the Institute of Heavy Rain, China Meteorology Administration (CMA), Wuhan, from AremV2.1, AremV2.3 to the current AremV3.0. Many years of forecast results show that the AREM model has a strong ability for spatial and temporal distribution forecasting, and can more accurately give the distribution of the rain zone in the rainy season in South China and the Yangtze River. The main centre and the intensity of precipitation are close to the actual situation; it is also a good model to predict the occurrence, development and the end time of precipitation; furthermore it can correctly reflect the space-time evolution characteristics of summer precipitation in southern China. So AremV3.0 was selected for testing the precipitation forecast and precipitation ensemble forecast ( $\mathrm{Yu} \mathrm{Ru}$-cong 1989, Cui Chun-guang et al. 2007).

\subsection{The scheme and analysis of model forecast and ensemble forecast}

The rainstorm from 21 to 23 July 2008 in the research area (referred to as " 08.7 " below), was selected to test the precipitation forecast and precipitation ensemble forecast. During the rainstorm, the accumulated precipitation in the research area amounted to $150 \mathrm{~mm}$ within $48 \mathrm{~h}$ and the water level of the reservoir suddenly rose by $2 \mathrm{~m}$ in the evening of 23 July (Chen Zheng-hong 2009, Cui Chun-guang and Peng Tao 2010)

Here, we adopted the AREM model to make the forecast test. The start time of the controlled trial (CTL) forecast was 722 08:00 in 2008 (Beijing Time), using the $6 \mathrm{~h}$ interval NCEP reanalysis data as model initial and lateral boundary conditions, with no nesting, mode resolution of $37 \mathrm{~km}$ and integration time of $36 \mathrm{~h}$. The forecast test show that the results are more consistent with the observations in spatial distribution, and is relatively stronger than the observation in precipitation intensity. Overall, the forecast result is ideal and can be used as flood forecast test.

Based on the controlled trial forecast test, we select a random perturbation scheme, and randomly perturb the wind field, temperature field, vapour field and ground pressure field on the control forecast analysis field (its perturbation amplitude is the $12 \mathrm{~h}$ forecast error of the respective different mode variable), and then multiply with different random numbers to obtain the 
disturbance field. The test produced a total of five group perturbations, combining the control forecast analysis field with random perturbation fields. Denoted as the N1, N2, N3, N4, N5 programs, including the controlled trial forecast, there is a total of six members of the ensemble forecast of precipitation (Li Jun and Du Jun 2009, Li Jun et al. 2010).

According to the forecast result of the six ensemble members, the cumulative average area rainfall in $36 \mathrm{~h}$ can be obtained (Table 1). The results show that the precipitation in the next $36 \mathrm{~h}$ in the research area will vary from 59.02 to $105.14 \mathrm{~mm}$, and will have a stronger precipitation process. Within the six members, the forecast results of CTL, N2 and N3 are close to the observations and the mean precipitation reaches the rainstorm magnitude. If the results are input to the hydrological model to make the hydrological forecasting, we can calculate the corresponding peak flow and the flood peak arrival time and get more hydrological forecast information.

Table 1 The future $36 \mathrm{~h}$ precipitation of observation and ensemble prediction after 08:00 22-7-2008 in ZhangHe basin,

\begin{tabular}{|c|c|c|c|c|c|c|c|c|}
\hline \multirow[t]{2}{*}{$\mathrm{CP}$} & \multirow[t]{2}{*}{ OBS } & \multicolumn{7}{|c|}{ Ensemble forecast scheme } \\
\hline & & CTL & N1 & N2 & N3 & N4 & N5 & Mean \\
\hline $\mathrm{R} 36 / \mathrm{mm}$ & 99.97 & 104.67 & 68.02 & 94.02 & 105.14 & 59.02 & 70.85 & 83.62 \\
\hline \multicolumn{9}{|c|}{$\mathrm{CP}$, Cumulative precipitation; OBS, observation precipitation. } \\
\hline
\end{tabular}

\section{THE EXPERIMENT}

\subsection{Hydrological model selection}

Based on the humid climate characteristics of the research area, the Xin'Anjiang model was selected as the hydrological forecasting model; it is widely used at home and abroad. The model adopts the concept of storage runoff and Muskingum afflux, and has the characteristics of unit, water sources and afflux phase, with a simple structure and few parameters which have a clear physical meaning and high accuracy. The model divides the whole catchment into sub-catchments, and then the model makes the runoff and afflux calculation in each unit, and gets the flux at the outlet of the unit, and then calculates the flood in the river, summing all the afflux and to give the whole flux of catchments (Zhao Ren-jun, 1984).

\subsection{The application of precipitation forecast to the hydrological forecast}

During the test, we use rain stations or radar quantitative precipitation estimation technology to get $24 \mathrm{~h}$ observation rainfall before the forecast start time, and then use AREM numerical models to obtain rainfall in the future 6, 12, 18, 24 and 36 hours, and input them to the Xin' Anjiang model to make the flood forecast, producing the flood process curves shown in Fig. 2. Based on the standard for hydrological information and hydrological forecasting, the relative error and the time difference of the flood peak is adopted to evaluate the forecast result (SL25-, 2000).the results are shown in Table 2. From the Fig. 2, we found that the forecast flood process curve has obvious differences with the observations when we do not consider the future precipitation (Fig. 2(a)). If we consider the precipitation in 6 hours future, the forecast flood process curve still has a bigger difference because the main rain process does not occur (Fig. 2(b)). Using the 12 hour future data, we found that the forecast curve changes; the curve weakens first, and then rises, but certain disparities with observation still exist (Fig. 2(c)). With 18 hours of future precipitation, the main precipitation process is basically completed; the forecast curve rises obviously, and starts to be close to observation, but still has certain disparities (Fig. 2(d)). With 24 and 36 hours future precipitation there is no obvious difference; the rainfall process ends basically, so the forecast curve is close to the observation curve (Fig. 2(e)-(f)). The results show that: under the conditions that the model forecast results are accurate, forecasts which consider future rain have obvious effects on the flood forecast result; the relative error can be reduced by $40 \%$. But when the time of prediction continues, the rainfall process tends to end and the impact of considering the rain in future on the flood forecast results will tend to decline. 


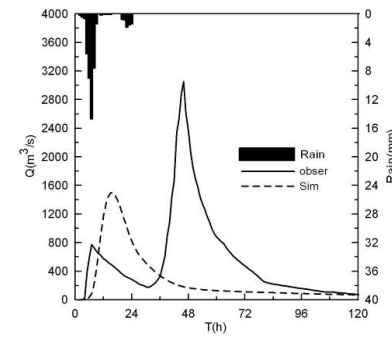

a, without precipitation in future

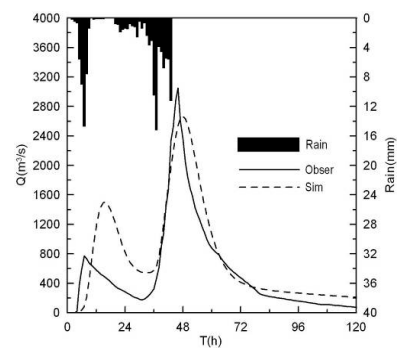

$\mathrm{d}$, with precipitation in $18 \mathrm{~h}$ future

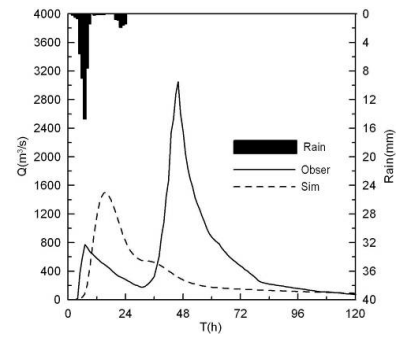

$\mathrm{b}$, with precipitation in $6 \mathrm{~h}$ future

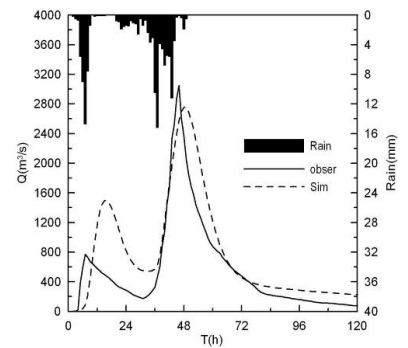

e, with precipitation in $24 \mathrm{~h}$ future

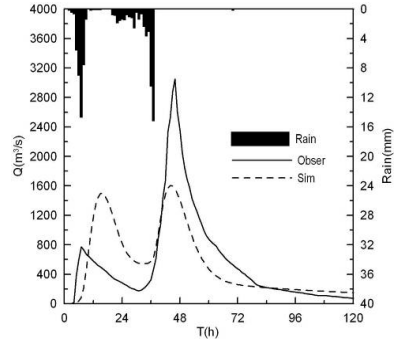

$\mathrm{c}$, with precipitation in $12 \mathrm{~h}$ future

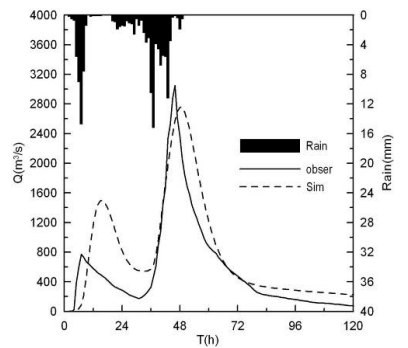

$\mathrm{f}$, with precipitation in $36 \mathrm{~h}$ future

Fig. 2 The contrast of flood flow process with different rain in forecast period in the 08.7 process.

Table 2 The contrast of flood flow process with different rain in the forecast period.

\begin{tabular}{llllll}
\hline Prediction time & $\begin{array}{l}\text { Future } \\
\text { precipitation }(\mathrm{h})\end{array}$ & $\begin{array}{l}\text { Observation } \\
\text { flood peak }\left(\mathrm{m}^{3} / \mathrm{s}\right)\end{array}$ & $\begin{array}{l}\text { Simulation flood } \\
\text { peak }\left(\mathrm{m}^{3} / \mathrm{s}\right)\end{array}$ & $\begin{array}{l}\text { Relative error } \\
(\%)\end{array}$ & $\begin{array}{l}\text { Time difference } \\
(\mathrm{h})\end{array}$ \\
\hline $7-2208: 00$ & 0 & 3050 & 1496 & 50.95 & 30 \\
& 6 & & 1496 & 50.95 & 30 \\
& 12 & & 1604 & 47.41 & 2 \\
& 18 & 2665 & 12.61 & 2 \\
& 24 & 2755 & 9.67 & 2 \\
& 36 & 2755 & 9.67 & 2 \\
\hline
\end{tabular}

\subsection{The application of ensemble precipitation forecast in hydrological forecast}

We take the 0800 am on 22 July 2008 as the start time of the flood forecast and obtain the antecedent $24 \mathrm{~h}$ observed precipitation and make it as the initial precipitation of the hydrological model, and then based on the results of AREM Ensemble forecasting (CTL, N1, N2, N3, N4, N5), the hourly precipitation forecast results for the next $36 \mathrm{~h}$ are obtained, and input to the Xin' Anjiang hydrological model for the flood forecasting test. The flood process curves are shown in Fig. 3. The relative error and the time difference of the flood peak are adopted to evaluate the forecast result, and the results are shown in Table 3.

From the Fig. 3, the simulated flood flow process curve is more consistent with the observation when using the precipitation forecast results of CTL, N2, N3 members in the hydrological model (Fig. 3(a), (c), (d)). The simulated curve has large difference with the observations when using N1, N4, and N5 in the hydrological model (Fig. 3(b), (e), (f)). The main reason is that the forecast precipitation of the CTL, N2, N3 members is close to the observed precipitation, the error is about approximately 5\%. While the forecast precipitation of N1, N4, N5 members reach rainstorm magnitude, it still differs from observed (by $30-40 \mathrm{~mm}$ ), and the error is about 30 to $40 \%$. From Table 3, the calculated peak flow is $2755 \mathrm{~m}^{3} / \mathrm{s}$. Using the next $36 \mathrm{~h}$ observed precipitation in the hydrological model, the relative error is $9.67 \%$. The flood peak flow range of 1491 to $2944 \mathrm{~m}^{3} / \mathrm{s}$ can be calculated when using the ensemble precipitation forecast results of the AREM, and the relative error ranges from 51.11 to $3.48 \%$; the forecast maximum of flood peak is almost the same as observed. 


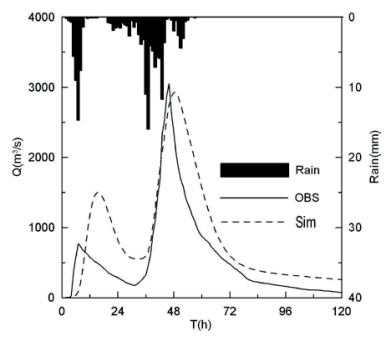

(a) CTL

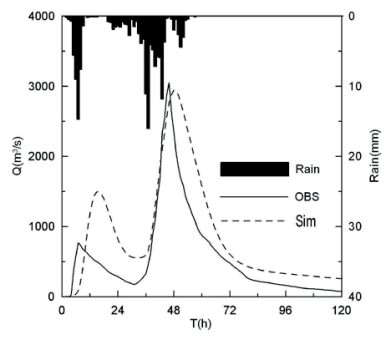

(d) $\mathrm{N} 3$

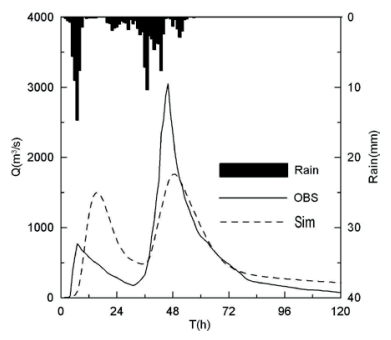

(b) $\mathrm{N} 1$

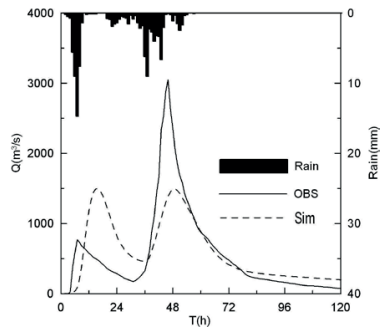

(e) N4

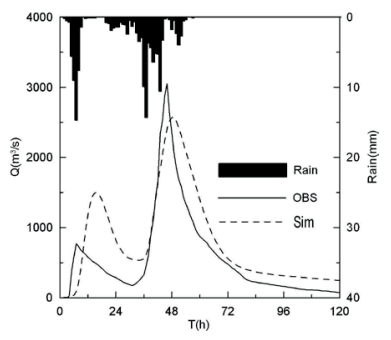

(c) $\mathrm{N} 2$

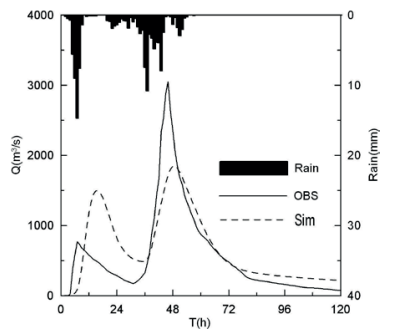

(f) N5

Fig. 3 The flood flow process on the different Ensemble Precipitation prediction of (a) CTL, (b) N1, (c) N2, (d) N3, (e) N4 and (f) N5 in the 08.7 process.

Table 3 Evaluation of flood forecasts with different precipitation test schemes in 08.7 processes.

\begin{tabular}{llllll}
\hline Prediction time & $\begin{array}{l}\text { Observation flood } \\
\text { peak }\left(\mathrm{m}^{3} / \mathrm{s}\right)\end{array}$ & Test scheme & $\begin{array}{l}\text { Simulation flood } \\
\text { peak }\left(\mathrm{m}^{3} / \mathrm{s}\right)\end{array}$ & $\begin{array}{l}\text { Relative error } \\
(\%)\end{array}$ & $\begin{array}{l}\text { Time difference } \\
(\mathrm{h})\end{array}$ \\
\hline 7-22 08:00 & 3050 & OBS & 2755 & 9.67 & 2 \\
& & CTL & 2927 & 4.03 & 2 \\
& & N1 & 1761 & 42.26 & 3 \\
& & N2 & 2571 & 15.70 & 2 \\
& & N3 & 2944 & 3.48 & 2 \\
& N4 & 1491 & 51.11 & 3 \\
& N5 & 1849 & 39.38 & 3 \\
\hline
\end{tabular}

\section{CONCLUSIONS}

Precipitation is very important information in flood forecasting. Precipitation has a direct impact on the flood forecasting accuracy, but the uncertainty of input information into hydrological models seriously influence hydrological forecast precision. Precipitation ensemble prediction can provide more quantitative forecast information for hydrological forecasting, and help to enrich hydrological forecast information. Based on other studies, the paper takes the floods in 2008 in ZhangHe basin in Hubei province, China, as an example, uses the AREM model forecast and its ensemble forecast results as precipitation in the forecast period as input to the hydrological model, and tests the impact on flood forecast results:

(1) During the flood forecast, considering precipitation in the forecast period gives obvious improvements of up to $40 \%$, and it can make forecast of the flood process in advance during the whole rain process. With the development of the precipitation process, when the precipitation declines or the main process ends, the future precipitation has less impact on the flood forecast result.

(2) Precipitation ensemble prediction can provide more precipitation forecast information than definite forecast, and help to enrich the input information of the hydrological forecast. Thus the shape and timing of the flood peak can be obtained more reliably, making the sole definite forecast result transform to possible forecast. 
(3) Although that the precipitation in the forecast period is considered, there still is a difference between the flood forecast result and actual observation. The differences show that the forecast accuracy of the precipitation needs to be improved further.

(4) The impact of the forecast precipitation on the flood forecast have been noted by many hydrologists and meteorologists, and modern weather forecasting technology provides good technical support to offer the precipitation in the forecast period for flood forecasting. With the improvement of the spatial and temporal resolution and prediction accuracy of the numerical model forecast, it will have wide application potential in hydrology.

Acknowledgements The study is supported by the National Natural Science Foundation of China (41205086) and the National Key Basic Research and Development Plan of China (2013CB430206) the State Key Laboratory of Severe Weather, Chinese Academy of Meteorological Sciences (2014LASW-B09).

\section{REFERENCES}

Chen Zheng-hong et al. (2009) Characters and effects of rainstorm and flood disasters on July 20-23, 2008 in Hubei Province. Torrential Rain and Disasters 28(4), 345-348

Cui Chun-guang et al. (2007) Development and application of the advanced regional eta-coordinate numerical heavy-rain prediction model system in China. Advances in Geosciences 6, 23-33.

Cui chun-guang et al. (2010) The Flood Forecast Test on QPF Coupling with Hydrological Model in Flood Season in medium and small catchment. Meteorological Monthly 36(12), 56-61.

Gong Ying et al. (2007) Evaluation and analysis of AREM prediction products in flood season of 2005 . Meteorological Monthly 33(1), 87-93.

Li Chaoqun et al. (2006) Application of short-term quantitative precipitation forecasts in flood forecasting in Geheyan Reservoir basin. Water Resources and Power 24(4), 31-35.

Li Jun et al. (2007) Precipitation forecast experiments of a meso-scale numerical model AREM. Engineering Journal of Wuhan University 40(1), 13-16.

Li Jun, et al. (2009) Experiments of perturbing initial conditions in the development of mesoscale ensemble prediction system for heavy rainstorm forecasting. Plateau Meteorology 28(6), 1365-1375

Li Jun, et al. (2010) Precipitation verifications to an ensemble prediction system based on AREM. Torrential Rain and Disasters 29(1), 30-37.

Li Yan et al. (2008) Application of Ensemble Streamflow Prediction (ESP) to medium and long term water resources prediction. Hydrology 28(1), 25-30.

Yang Wen-fa. (2003) The primary research on coupling application in precipitation and flood forecast. The investigation of water resource $24(1), 38-40$.

Yu Ru-cong (1989) The design of limited district numerical forecast model in cragged landform. Atmospheric Sciences 13(2), 139-149.

Zhao Ren-jun (1984) Hydrological Simulation in Basins. WaterPower Press. Beijing. 